\title{
Sound wave propagation in weakly polydisperse granular materials
}

\author{
O. Mouraille, S. Luding \\ NSM/DCT/TUDelft, Julianalaan 136, 2628 BL Delft, Netherlands
}

\begin{abstract}
Dynamic simulations of wave propagation are performed in dense granular media with a narrow polydisperse size-distribution and a linear contact-force law. A small perturbation is created on one side of a static packing and its propagation, for both $\mathrm{P}$ - and S-waves, is examined. A size variation comparable to the typical contact deformation already changes sound propagation considerably. The transmission spectrum becomes discontinuous, i.e., a lower frequency band is transmitted well, while higher frequencies are not, possibly due to attenuation and scattering.

This behaviour is qualitatively reproduced for (i) Hertz non-linear contacts, for (ii) frictional contacts, (iii) for a range of smaller amplitudes, or (iv) for larger systems. This proves that the observed wave propagation and dispersion behaviour is intrinsic and not just an artifact of (i) a linear model, (ii) a frictionless packing, (iii) a large amplitude non-linear wave, or (iv) a finite size effect.
\end{abstract}

Key words: DEM simulation, granular matter, sound propagation, friction, non-linear phenomena

PACS: 43.25.-x, 46.10+z, 02.70 Ns.

\section{Introduction}

The stress-wave (sound) propagation through dense granular matter is the superposition of many complex phenomena, which are caused by the discrete, inhomogeneous, anisotropic and dissipative structure of this class of materials on the microscopic, contact scale $[1,2,3,4,5]$. Non-linear contacts $[6,7]$, forcechains [8], frequency-dependence $[9,10]$ and also scattering and attenuation in other "particle type" materials [11] are reported. Ballistic pulse propagation

Email address: 0.J.P.Mouraille@tudelft.nl, s.luding@tudelft.nl (O. Mouraille, S. Luding). 
can co-exist with a slower multiply scattered, coda-like signal $[4,12]$, and the stress- and frequency-dependence of the wave propagation features are subject of ongoing discussion $[3,4]$ in static and shaken packings as well.

A continuum description of granular materials is generally needed in field applications (like oil recovery) due to the huge system-size. On this macroscopic scale, the properties of such waves are strongly affected by phenomena like attenuation, scattering, and dispersion [4]. Starting from simulations at the particle level, which are necessary to gain insight into the role of the micro-parameters, a "micro-macro" transition [13,14] can provide a continuum, macroscopic description (at least for quasi-static deformations). The ultimate goal is to find micro-based macroscopic constitutive relations, superior to the empirical ones typically used, also for dynamic and possibly non-linear deformation and propagation modes $[3,4,16]$.

The use of a three-dimensional discrete element method (DEM) [7,17,18,19,20,21] allows the study of the role of various micro-parameters in detail. Except for a regular crystal structure of exactly equal sized particles, geometrical (and hence contact) disorder is intrinsic to granular systems. The wave propagation properties are examined in the following, starting from a regular structure and slowly increasing the amount of disorder involved. The system size and the amplitude are varied as well as the non-linearity and friction in order to understand their effect on the wave-propagation characteristics.

\section{Simulation setup}

\subsection{DEM Model}

The elementary units of granular materials are mesoscopic grains, which deform locally under the forces/stresses acting at their contacts. Since the realistic modeling of the internal deformation of the particles is too expensive, the normal interaction force is only related to the "overlap" of two (spherical) particles, while the tangential forces involve an elastic and a frictional element. If the sum of all forces, $f_{i}$, acting on particle $i$, either from other particles, from boundaries or from external forces, is known, the problem is reduced to the integration of Newton's equations of motion for the translational and rotational degrees of freedom:

$$
m_{i} \frac{\mathrm{d}^{2}}{\mathrm{~d} t^{2}} \boldsymbol{r}_{i}=\boldsymbol{f}_{i}, \quad \text { and } \quad I_{i} \frac{\mathrm{d}}{\mathrm{d} t} \boldsymbol{\omega}_{i}=\boldsymbol{t}_{i}
$$


with the mass $m_{i}$ of particle $i$, its position $\boldsymbol{r}_{i}$, its moment of inertia $I_{i}$, its angular velocity $\boldsymbol{\omega}_{i}$, and the total torque $\boldsymbol{t}_{i}$. Note that the above equation is only valid for isotropic bodies, like spheres as also used in the following $[17,20]$.

A simple linear spring is used for the normal force $|f|=k \delta$, with the contact stiffness $k\left(=10^{5} \mathrm{~N} / \mathrm{m}\right)$, corresponding approximately to the stiffness of a Plexiglas bead (detailed in section 2.3). If the overlap is positive, the force acts, if the overlap is negative the force is $f \equiv 0$. For the simulations where friction is active, the force in the tangential direction, $\boldsymbol{f}_{t}$, is implemented in the spirit of Ref. [18], where a tangential spring (with stiffness $k_{t}=0.2 k$ ) was introduced to account for static friction [17]. The tangential contact forces are related to the normal forces via Coulombs law, i.e., $f^{t} \leq \mu f^{n}$, with $\mu=0.5$ for both sliding and sticking. In some simulations, a non-linear spring model (according to Hertz) was used, with the normal force $|f|=k_{H}\left(\delta^{3 / 2}\right)$, with $k_{H}=4.510^{7} \mathrm{~N} / \mathrm{m}^{3 / 2}$.

\subsection{Particle packing}

The geometrical configuration considered in this study is a dense, static packing of grains, with radii $a_{0}(=0.001 \mathrm{~m})$, arranged in a Face Centered Cubic (FCC) structure (with density, i.e., volume fraction of $\nu \approx 0.74$ ), where square layers in the $x$ - $y$-plane $(4 \times 4$ particles) are stacked in the $z$-direction (200 layers), see Fig. 1 for a snapshot of a part of the system. The larger systems examined are double-sized in the cross-section $(8 \times 8$ particles). This regular, ordered system is rather thin but elongated in the $z$-direction in order to allow the wave to propagate for some time, before it hits the wall on the opposite side. Each particle has four contacts inside each square-layer, and eight with particles in the neighbouring layers (4 each), corresponding to a coordination number of $C=12$. All particles have the same overlap $\bar{\delta} / a_{0} \approx 10^{-3}$ and (due to periodic boundaries) see the same environment (except those in the first and last mobile layer).

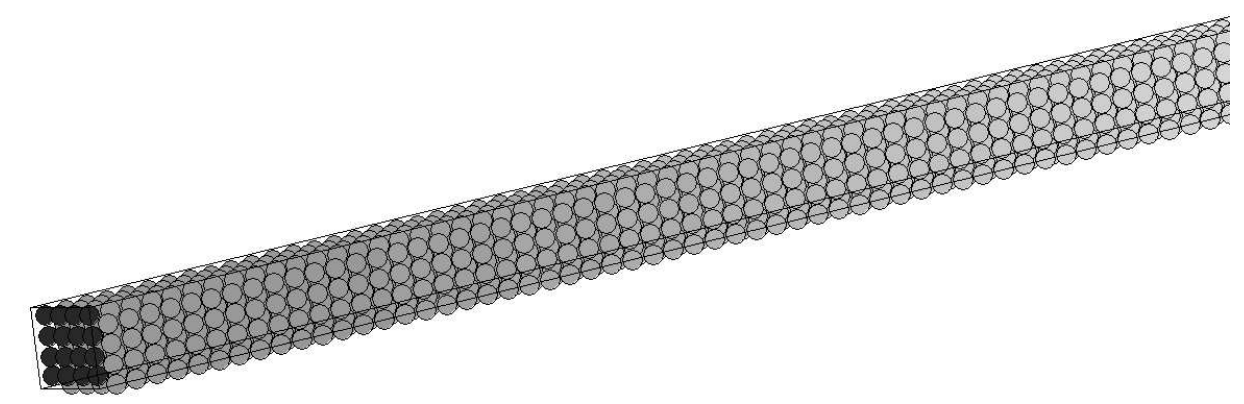

Fig. 1. FCC-structured packing, thin $(4 \times 4$ periodic) in the $x$ - and $y$-directions, and elongated (non-periodic) in the $z$-direction; the black particles indicate the "zero-th" layer of fixed particles that make up the $z=z_{0}$ boundary. 
Polydisperse packings are obtained by randomly changing the particle sizes according to a narrow size distribution, which is homogeneous, centered at $a_{0}$, and has a width $2(\Delta a)$ of the order of the mean overlap $\bar{\delta}$ and then allowing the system to relax to a new, slightly disordered equilibrium configuration with the same volume. More precisely four different cases, with $(\Delta a)=0, \bar{\delta} / 2, \bar{\delta}$ and $2 \bar{\delta}$ are compared. Note that the polydisperse systems conserve their original FCC structure at the grain scale (almost), since the radius variation is much smaller than the particle sizes, however, strong differences are observed at the contact level. This is because, a radius variation of $(\Delta a)=2 \bar{\delta}$, represents only a small change of $0.2 \%$ at the grain level but a large change of $200 \%$ at the contact level. This has a direct influence on the coordination numbers, which drop down from $C=12$ to $C \approx 11.99,11.22$, and 9.97, for the three $(\Delta a)$, respectively. For the last case this means a non-negligible reduction of about $17 \%$ of the total contacts of the monodisperse system. Since the effect of this "tiny" radius variation is so strong, polydispersity should be seen as related to the overlap rather than the particle size, as noted already in Ref. [22].

In order to examine the effect of more realistic contact models, one packing with $(\Delta a)=2 \bar{\delta}$ was relaxed with friction active, leading to an even smaller coordination number of $C \approx 9.64$. Another packing, also with $(\Delta a)=2 \bar{\delta}$ was relaxed using the Hertz contact-model, see section 2.1, so that, the coordination number was $C \approx 9.77$, somewhat lower than for the packing with the linear model $(C \approx 9.97)$. Note that all these packings are still much more coordinated and denser than the theoretical isostatic limit cases of $C=6$ and $C=4$ for frictionless and frictional packings, respectively.

\subsection{Wave agitation}

In order to agitate a plane wave in $z$-direction, an initial velocity $\boldsymbol{v}$ is given to all the particles that make up the source, i.e., the first $x$-y-layer with $z=$ $z_{1}$, in contact with the fixed particles at $z=z_{0}$. This velocity either points in $z$-direction, $\boldsymbol{v}=v_{z} \hat{\boldsymbol{z}}$, or in $x$-direction, $\boldsymbol{v}=v_{x} \hat{\boldsymbol{x}}$, creating a compressive $(\mathrm{P})$, or a $x$-polarized shear $(\mathrm{S})$ wave, respectively. If not mentioned, explicitly, $v_{z}=v_{x}=0.01 \mathrm{~m} / \mathrm{s}$ are used, which create a maximum particle displacement $u_{\text {max }} \approx 510^{-8} \mathrm{~m}$, during the simulations. Relating the layer distance $l_{z}=z_{1}-$ $z_{0}=\sqrt{2} a_{0}$ and the "contact duration" (Ref. [17]) $t_{c} \approx 2.0310^{-5} \mathrm{~s}$, leads to a velocity $v_{c}=l_{z} / t_{c} \approx 70.7 \mathrm{~m} / \mathrm{s}$. The chosen contact stiffness, $k\left(=10^{5} \mathrm{~N} / \mathrm{m}\right)$, as mentioned earlier, correspond to the stiffness of a Plexiglas bead. Meaning that this value is close to the stiffness of the non-linear spring model (using Plexiglas elastic constants), calculated at the packing overlap in the monodisperse case, here $\bar{\delta} / a_{0} \approx 10^{-3}$. At this overlap, the stiffness and the contact duration of a particle-particle collision appear to be of the same order for both linear and non-linear model. 


\section{Results}

In the following, the stress-time response to the initial perturbation is recorded at each layer with a large binning rate, $\Delta t=10^{-6} \mathrm{~s}$, in a time-window of $T=0.0015 \mathrm{~s}$, to be further analyzed for all systems described above in section 2.2. Note that even though the boundary conditions and the excitation method both influence the stress-time signal shape (data not shown), we only use the velocity-pulse method in this study for convenience; a detailed study of different pulse methods will be published elsewhere.

\subsection{Linear model}

\subsubsection{From order to weak disorder}

The stress-response - ten layers from the source - is plotted against time in Fig. 2 (Left) for the monodisperse and the polydisperse situation with $(\Delta a)=2 \bar{\delta}$ (for more details see Refs. [19,20,21]). Only these two last cases will be shown in this study, since the case $(\Delta a)=\bar{\delta} / 2$ resembles the monodisperse system, and the case $(\Delta a)=\bar{\delta}$ behaves similar to the case $(\Delta a)=2 \bar{\delta}$. Qualitatively, signals arrives later in the polydisperse system, which is consistent with a lower propagation speed (see Ref. [21]) due to the smaller coordination number, as compared to the monodisperse system. The signal amplitude is smaller in the polydisperse system, which indicates that some energy has been transferred (scattered) to other types of motion (waves of different nature, like shear waves, and/or with different propagation direction). Finally, the coda of the signal in the polydisperse packing is clearly irregular, while it is periodic in the monodisperse case.
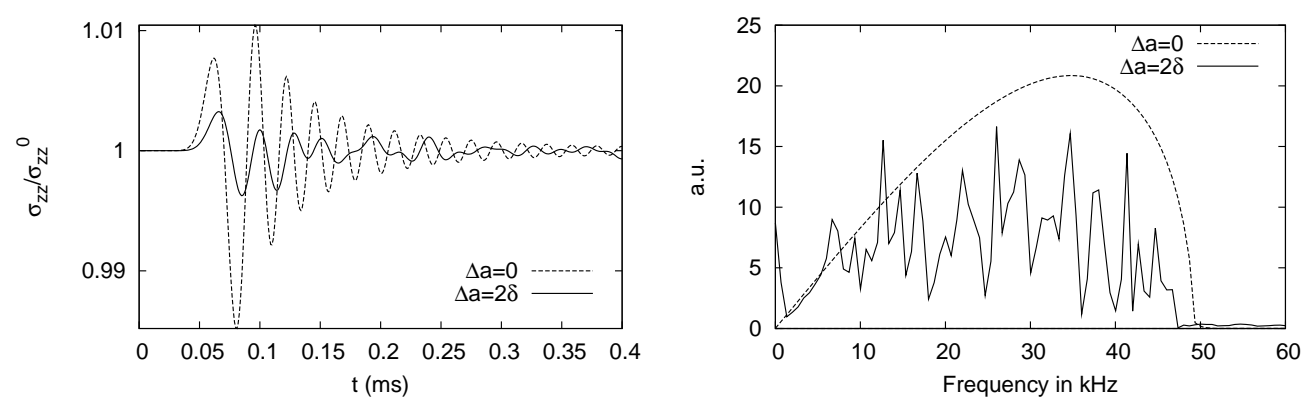

Fig. 2. (Left) Normal stress $\left(\sigma_{z z}\right)$ scaled by the equilibrium stress $\left(\sigma_{z z}^{0}\right)$ as function of time at a distance of 10 layers from the source for the monodisperse (dashed line) and a polydisperse (solid line) system. (Right) Fourier power spectrum of the two stress-time signals from the left figure.

In order to analyze the two signals in more detail, the frequency content is examined from the Fourier power spectrum in time, see Fig. 2 (Right). The 
monodisperse system shows a smooth spectrum, while in the polydisperse system, due to the disorder present at the contact level, some frequencies seem to be filtered stronger than others, i.e., the spectrum shows peaks and valleys.

In order to understand the propagation properties of the wave-components with different frequencies, the frequency power spectra are plotted (vertical) against the position in $z$-direction (horizontal) in Fig. 3 in grey-scale.

In the monodisperse case (Left), frequencies above about $49 \mathrm{kHz}$ are immediately attenuated, since the packing cannot transmit frequencies higher than the (largest) eigen-frequency of a single layer. From the third layer on, the range of frequencies present in the time signal is conserved during the propagation. Note that the bending observed, beginning at about 50 layers from the source, is only due to the limited time window of the collected data, i.e., for larger time windows, the bending starts later. This bending, top right, is consistent with a smaller propagation speed of higher frequency wave components.

In the polydisperse case (Right), the power spectrum shows a strongly reduced range of transmitted frequencies as the wave propagates away from the source. Frequency transmission over large distances is observed under about $8 \mathrm{kHz}$ and the higher frequencies (about $8-46 \mathrm{kHz}$ ), present near the source, decay exponentially (data not shown) between layers 20-40. Note that some special frequencies propagate deeper into the system than others, as the band around $35 \mathrm{kHz}$ (eigen-frequency of a double layer) for example.
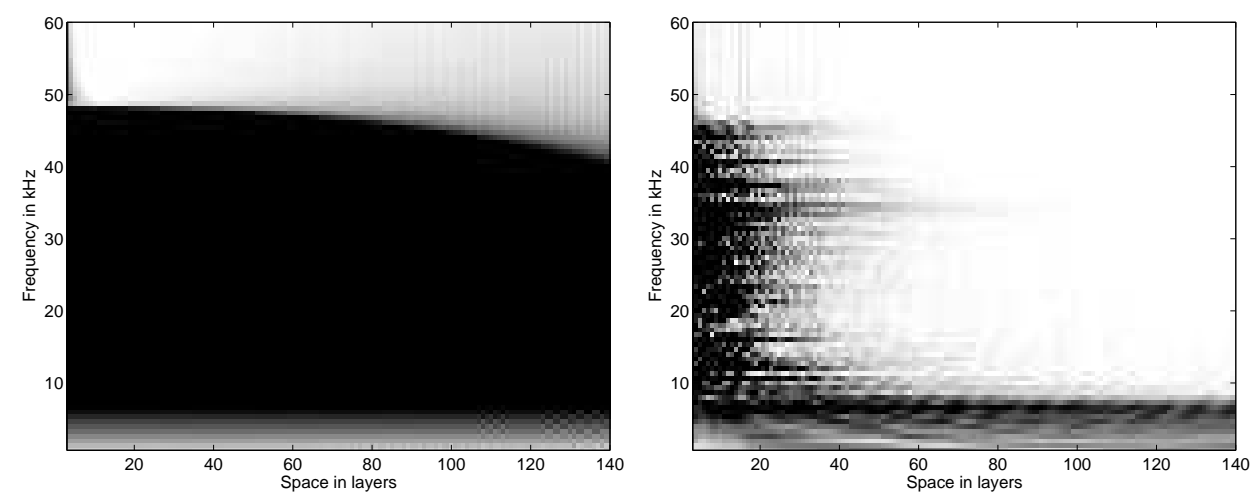

Fig. 3. Frequency-space diagram (darkness corresponds to the amplitude, absolute value, of the Fourier coefficients, black is large and white is small) for a P-wave propagating in $z$-direction in the monodisperse (Left) and the polydisperse (Right) packing from Fig. 2.

In order to understand possible relations between frequencies and wave-numbers, the two-dimensional Fourier transform in time and space is calculated from the same data as before. The dispersion relation in the monodisperse packing (angular frequency $\omega=2 \pi f$ versus angular wavenumber $\bar{k}=2 \pi k=2 \pi / \lambda$ ) 
has a perfect sine-shape $\omega(\bar{k})=\omega_{0} \sin \left(l_{z} \bar{k} / 2\right)$, with layer-width in $z$-direction, $l_{z}=\sqrt{2} a_{0}$, and P-wave speed, $V_{P}=\omega_{0}\left(l_{z} / 2\right) \approx 219 \mathrm{~m} / \mathrm{s}$, where $\omega_{0}=2 \pi f_{0}$, with $f_{0} \approx 49 \mathrm{kHz}$, see Fig. 4 and Refs. [20,21]. The minimal wave-length can be obtained from the maximum of the dispersion relation, $l_{z} \bar{k}_{\max } / 2=$ $\left(\pi l_{z}\right) / \lambda_{\min }=\pi / 2$, so that $\lambda_{\min }=2 l_{z}$.

For the polydisperse packing, with $(\Delta a)=2 \bar{\delta}$, the dispersion relation has generally smaller intensity, is smeared out much broader, and is more noisy, even though the sine-shape is still visible. Some band gaps appear, and one of them indicates the definite end of the linear behaviour (with a corresponding wave speed $V_{P} \approx 174 \mathrm{~m} / \mathrm{s}$ ) in the "low frequency/large wavelength" regime, somewhat smaller than for the monodisperse packing.

In order to analyze this peculiar dispersion relation, the mean wavenumber corresponding to a certain frequency, weighted only above a suited cut-off (here six times the average value of all Fourier-coefficients), is plotted on top of the dispersion relation as white dots in Fig. 4 (Right). The dots underline the linear regime, corresponding to the transmission-band at small frequencies, and also indicate the band-gaps in the cases where all Fourier coefficients corresponding to one frequency are smaller than the cut-off value. In the frequency range from $2-8 \mathrm{kHz}$ the dots are nicely linear, with a slope $V_{P} \approx 174 \mathrm{~m} / \mathrm{s}$. At higher frequencies, the dots (mean wave-numbers) are no longer falling on a straight line and band gaps as well as transmission bands appear. The clear band gap observed between $18-21 \mathrm{kHz}$ is also visible in Fig. 3 in so far that these frequencies penetrate least into the system.
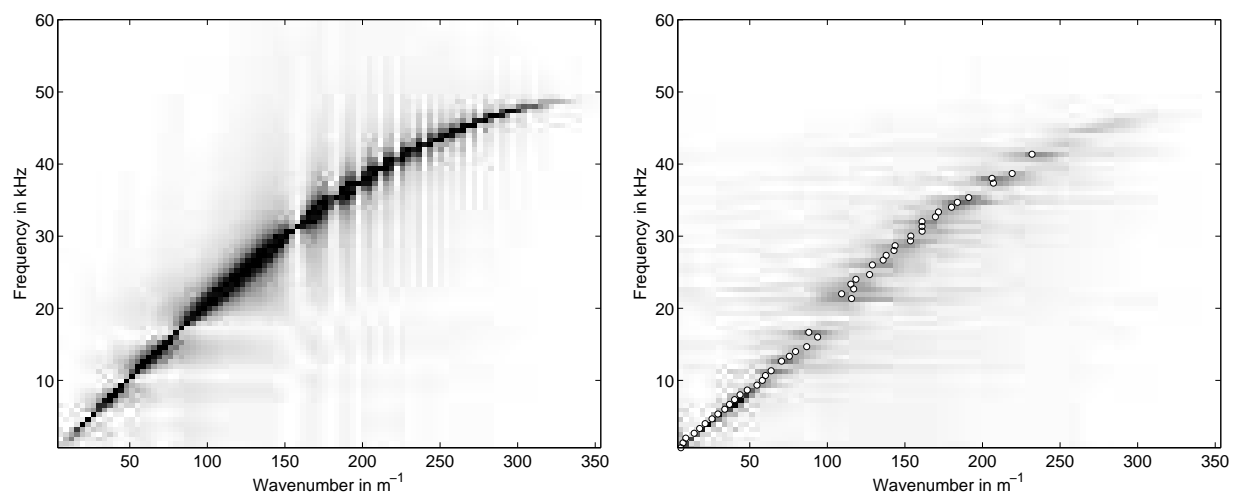

Fig. 4. Dispersion relation (darkness corresponds to the amplitude, absolute value, of the Fourier coefficients, black is large and white is small) for the same data as in Fig. 3 , for the (Left) monodisperse, and the (Right) polydisperse cases. The amplitude of the left sine is $49 \mathrm{kHz}$, while the slope for small frequencies and large wave-lengths, is $V_{P} \approx 219 \mathrm{~m} / \mathrm{s}$. The white dots in the right figure indicate the cut-off-weighted averaged wave-number per frequency, and their slope from $2-8 \mathrm{kHz}$ is $V_{P} \approx 174 \mathrm{~m} / \mathrm{s}$. 


\subsubsection{System size}

In order to study the influence of the system size on the wave propagation, simulations have been performed in systems with a four times larger area in the $x$-y-plane. The $z$-system size determines the smallest wavenumber (largest wavelength) present in the system: $k_{\min -\mathrm{z}}=1 / \lambda_{\max } \approx 1.77 \mathrm{~m}^{-1}$ with $\lambda_{\max }=$ $2 L_{z}$, where $L_{z}$ is the length of the system. The $x$-y-system-size $\left(L_{x}=L_{y}=\right.$ $8 a_{0}$ or $\left.16 a_{0}\right)$ corresponds to the wave-numbers $k_{\min -\mathrm{x}}=1 /\left(2 L_{x}\right) \approx 62.5 \mathrm{~m}^{-1}$ and $\approx 31.25 \mathrm{~m}^{-1}$ for the small and large systems, respectively. If the $x-y$ system size has an effect, one should expect special features at these wavenumbers, or the shift of features to smaller wave-numbers when the system size is increased. However, the results obtained with the larger system are much similar to those obtained with the smaller system, see Fig. 5. The band of transmitted frequencies is about the same, up to $8 \mathrm{kHz}$, and also the band-gap around $18 \mathrm{kHz}$ is obtained independent of the system-size. Most differences are observed in the higher frequency range, where the signal is more noisy anyway. Due to the better statistics, the plots look smoother and less noisy for the larger system. In conclusion, changing the system size by a factor of two should reveal finite size effects of the dispersion relation: transmission bands and band-gaps should be found at different wave-numbers, however, the size of the system perpendicular to the P-wave propagation direction has no visible effect.
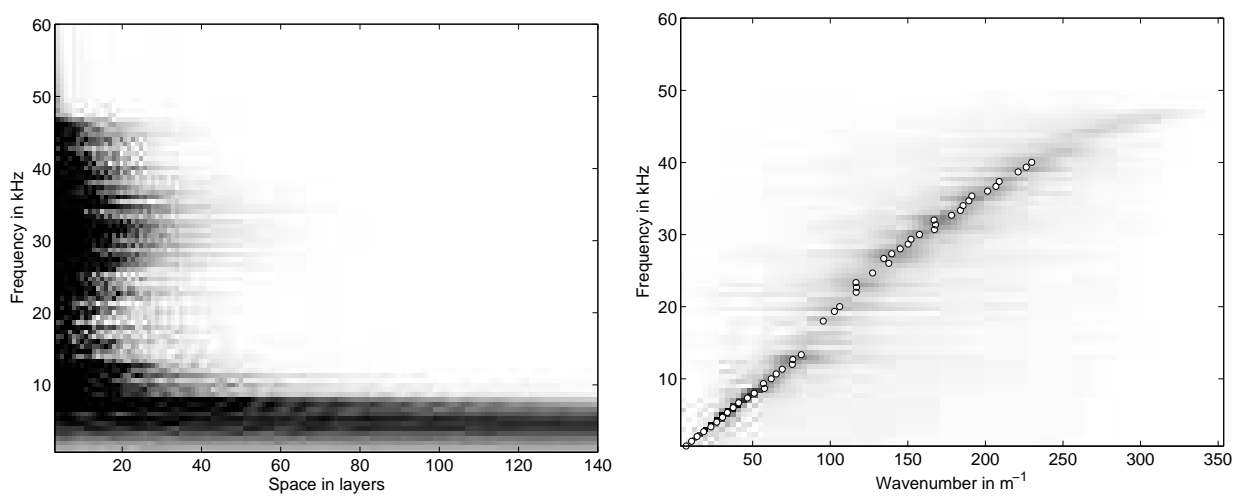

Fig. 5. Frequency-space diagram (Left) and dispersion relation (Right), (darkness corresponds to the amplitude, as in Figs. 3 and 4) of a P-wave for the polydisperse packing $(\Delta a)=2 \bar{\delta}$ with a larger section area of $8 \times 8$ particles. The slope of the dispersion relation leads to $V_{P} \approx 174 \mathrm{~m} / \mathrm{s}$ in agreement with the smaller system from Fig. 4.

\subsubsection{P- and S-waves}

The propagation of a S-wave in the (classical) monodisperse system was discussed in Ref. [20]. The ratio of $\mathrm{P}$ - to S-wave speeds was found to be $V_{P z} / V_{S z} \approx$ $\sqrt{2}$ - as expected from wave mechanics in crystals. The frequency range of 
possible S-waves ends at about $35 \mathrm{kHz}$ (eigen-frequency of a single particle in the $x$-direction) and, for a given wavenumber, the corresponding frequency is higher for the $\mathrm{P}$-wave than for the S-wave (data not shown).
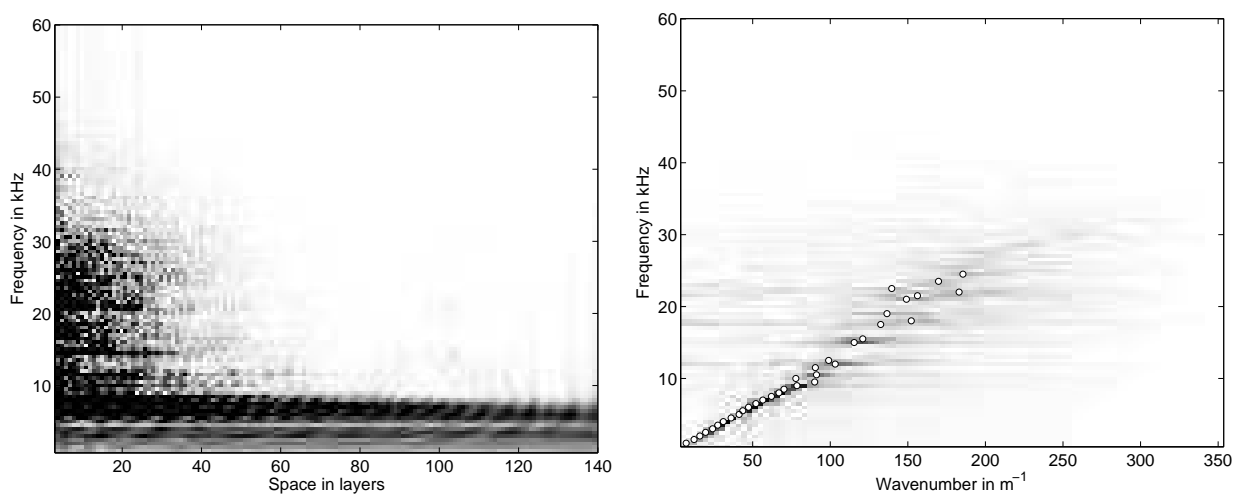

Fig. 6. Frequency-space diagram (Left) and dispersion relation (Right), (grey scale and symbols as in Fig. 5) of a shear wave for the polydisperse packing with $(\Delta a)=2 \bar{\delta}$.

The propagation of a S-wave in the polydisperse system, with $(\Delta a)=2 \bar{\delta}$, leads to the same dispersion behaviour as observed for the P-wave. The band of transmitted frequencies is also below $8 \mathrm{kHz}$ and the higher frequencies are limited by $35 \mathrm{kHz}$, like in the monodisperse case. More explicitly, the wave speeds are $V_{S} \approx 125 \mathrm{~m} / \mathrm{s}$ and $V_{P} \approx 175 \mathrm{~m} / \mathrm{s}$ for S- and P-waves, respectively, which is consistent with $V_{P} / V_{S} \approx \sqrt{2}$; thus no surprises are evidenced for S-waves in slightly polydisperse systems.

Finally, mode conversion was observed in the simulations, where S- to P-wave conversion appeared to be stronger than the P- to S-wave conversion. Since wave conversion contributes to the attenuation behavior (especially of the Swave), a future more detailed study is needed to analyze this issue further, including a possible frequency-dependence.

\subsection{Frictional packing}

When the particle contacts are frictional, i.e., a tangential spring, $k_{t}=0.2 k$, with Coulomb friction criterion, $\mu=0.5$, is added, see section 2.1 and Ref. [17], the overall frequency spectrum extends to higher frequencies, around $54 \mathrm{kHz}$, as a consequence of the tangential spring at the contact, which induces a higher particle (or layer) "eigenfrequency". However, the higher frequencies of the wave vanish earlier than in the frictionless case (between layers 1525), while the remaining low frequency band is broader and extends until about $12 \mathrm{kHz}$, see Fig. 7 (Left). The linear regime of the dispersion relation corresponds to a wave speed of $V_{P} \approx 200 \mathrm{~m} / \mathrm{s}$, i.e., somewhat slower than in the monodisperse case, but faster than in the polydisperse, frictionless case. 

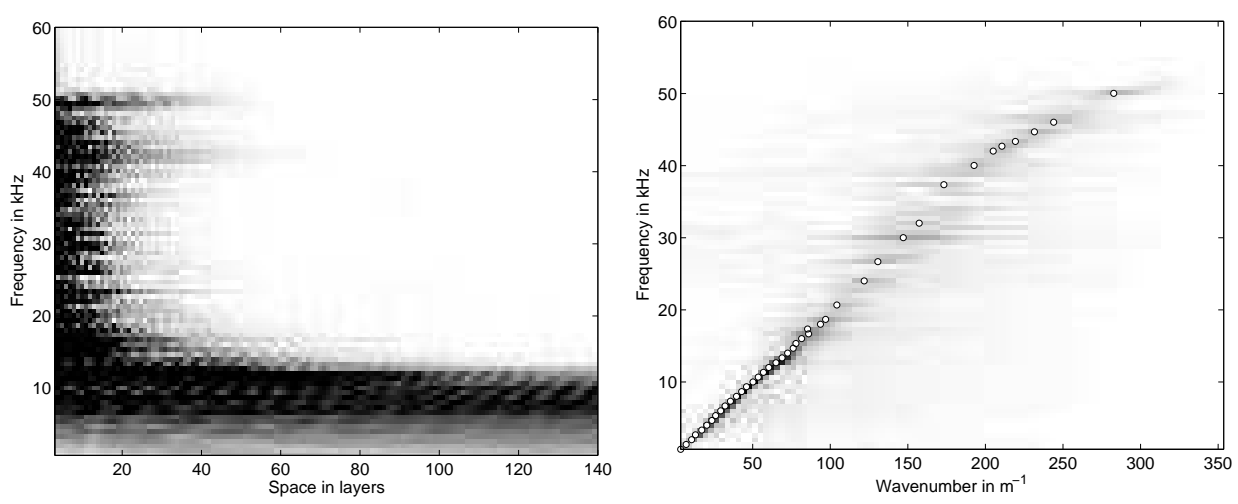

Fig. 7. Frequency-space diagram (Left) and dispersion relation (Right), (grey-scale and symbols as in Fig. 5), for a P-wave in the polydisperse packing $(\Delta a)=2 \bar{\delta}$ with friction.

The presence of friction, see Fig. 7, seems to extend or stabilize the linear dispersion regime for $\mathrm{P}$-waves. The non-linear, non-propagating regime with friction, is characterized by a relatively weaker signal. This might be due to the fact that in addition to the geometrical dispersion, which is also present in the frictionless case, friction enhances the compressive- to shear-mode conversion and also activates rotational modes to which part of the energy is lost. For shear waves in the monodisperse packing, in Ref. [20], an additional branch (rotational modes) in the dispersion relation was found (data not shown here), however, for the polydisperse packing $(\Delta a)=2 \bar{\delta}$ this could not be confirmed - thus remaining an issue for future studies.

\subsection{The Hertz contact model}

As it is reported in Refs. [7,24], the choice of the contact model has an influence on the frequency-dependent response of the system. Therefore, a simulation with another packing, prepared with the Hertz contact model, is performed. Qualitatively, the same wave propagation characteristics is observed as for the linear model. However, as consequence of a different initial state and due to the different nature (non-linear) of the contact model, the results obtained are quantitatively different. Due to a somewhat lower coordination number, $C \approx 9.77$, and thus some decreased packing stiffness in the $z$-direction, the wave travels a little slower, both for the $\mathrm{P}$-wave, $V_{P} \approx 156 \mathrm{~m} / \mathrm{s}$, see Fig. 8 , and for the S-wave $V_{S} \approx 106 \mathrm{~m} / \mathrm{s}$ (data not shown). Whether the ratio, $V_{P} / V_{S} \geq \sqrt{2}$, has a meaning, cannot be evidenced from our data.

A more detailed study of the shape of the wave-front and coda, e.g., under different stress conditions is far from the scope of this study. 

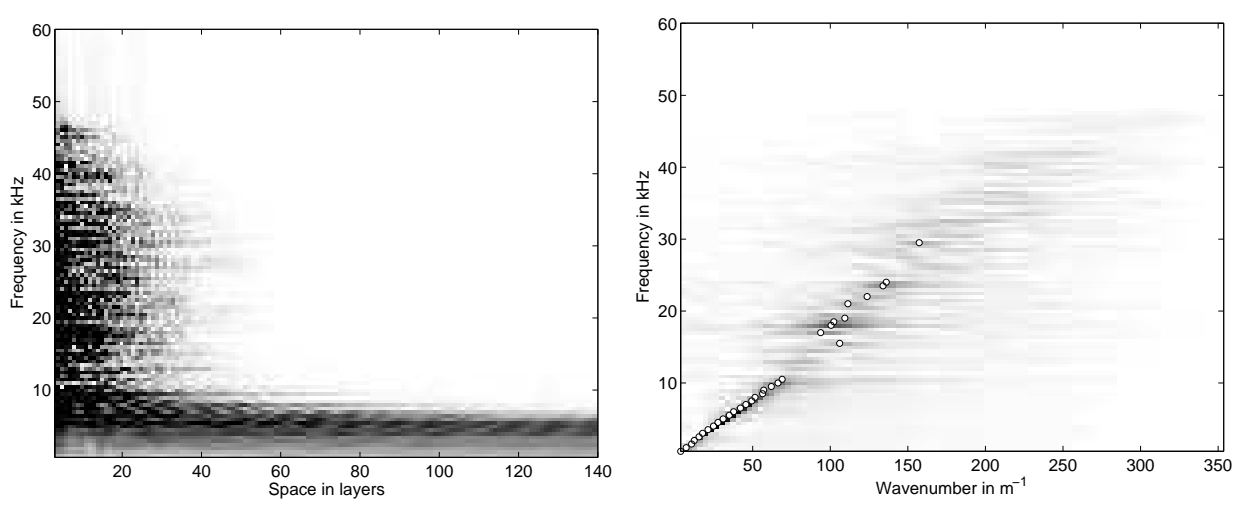

Fig. 8. Frequency-space diagram and dispersion relation (grey-scale and symbols as in Fig. 5), for a polydisperse packing with $(\Delta a)=2 \bar{\delta}$ and the Hertz contact model.

\subsection{Discussion of non-linearity and disorder}

The attenuation of frequencies, when the wave is propagating in a slightly polydisperse system, is a consequence of the change of the geometry and the corresponding material properties. The polydisperse systems are characterized by two main features: (i) a local (contact level) geometrical disorder, and (ii) the presence of clapping contacts, opening and closing while a wave is passing by.

This strong non-linearity at the contact level is specific to granular materials. It has, according to, Ref. $[25,16]$, its signature in the frequency content of the wave, closing contacts can generate high frequency signals, but opening contacts also interfere. In order to study the influence of the clapping contacts on the wave-propagation, in parallel to the polydisperse simulation from Figs. 3 (Right) and 4 (Right), two additional simulations have been performed. Everything was identical, only a 10 and 100 times smaller initial velocity $v_{z}$ was used, leading to less clapping contacts. In the case of the smallest velocity, only two contacts are clapping in the whole system, while for the largest velocity it is about 20 contacts $(0.1 \%)$.

The analysis of the frequency-space diagrams of these simulations, shows no qualitative difference for the dispersion, as compared to the previous simulations. The smaller $v_{z}$, the larger are (relatively) the lowest frequency and lowest wavenumber Fourier coefficients, so that the cut-off averaging can be applied in the range $f>3 \mathrm{kHz}$ and $k>12 \mathrm{~m}^{-1}$ only. The corresponding cut-offs used are proportional to the velocity amplitude and lead to cut-off weighted wave-numbers per frequency that are slightly decreasing with the initial velocity. However, this effect is very weak (comparable to the spacing between the data) and the dispersion curve (dots) always remains below the envelope of the monodisperse case. Thus, even though clapping contacts contribute to the non-linear response of the system, they do not play a key role in the observed attenuation of the higher frequency bands, given the present 
conditions (small amplitude $u_{\max } / \bar{\delta} \approx 510^{-2}$ with only $0.1 \%$ of clapping contacts).

Having reduced the clapping contact non-linearity by using smaller amplitudes and also having used linear as well as non-linear contact laws, the conclusion is that the geometrical disorder at the contact level is responsible for the attenuation of the higher frequencies of the wave in weakly polydisperse packings. Since more strongly disordered (polydisperse or not) packings would imply this same local (contact level) geometrical disorder, similar results are expected for such, more realistic, packings - as to be studied in the future.

\section{Summary and Conclusions}

Wave propagation was examined in three-dimensional ordered (crystal) packings with monodisperse, and with slightly polydisperse packings of spheres. The later involve contact level geometrical disorder, but are still close to a crystal structure. Compressive (P) and shear (S) waves were examined with and without friction; the system size and the initial agitation amplitude were varied and a non-linear Hertz type contact law was applied.

In all disordered cases (with size variation comparable to or larger than the typical contact deformation), a low frequency band transmits waves well, while most higher frequencies are attenuated or scattered within several tens of layers. Disorder (less contacts) leads to a reduced propagation speed of the wave, whereas friction (due to tangential elasticity) leads to an increased wave speed - and also to a broader transmission band. Also, the confining pressure, directly related to the static deformation of the contacts, should have an influence on the results and will be analyzed in a future study.

Having started with monodisperse situations and slowly increasing polydispersity, the natural next step is to confirm these observations also in the case of full disorder and wider size-distributions. The interesting transmission behavior in such more realistic granular packings can then be studied concerning friction, cohesion and rotational degrees of freedom.

\section{Acknowledgements}

This work is part of the research program of the "Stichting voor Fundamenteel Onderzoek der Materie" (FOM), financially supported by the "Nederlandse Organisatie voor Wetenschappelijk Onderzoek" (NWO) and the Stichting Shell Research. 


\section{References}

[1] C.-h. Liu and S. R. Nagel. Sound in sand. Phys. Rev. Lett., 68(15):2301-2304, 1992.

[2] C. S. Chang and J. Gao. Nonlinear dispersion of plane-wave in granular media. Int. J. Non-Linear Mech., 30(2):111-128, 1995.

[3] S. R. Hostler and C. E. Brennen. Pressure wave propagation in a shaken granular bed. Phys. Rev. E, 72:031304, 2005.

[4] X. Jia. Codalike multiple scattering of elastic waves in dense granular media. Phys. Rev. Lett., 93(15):154303, 2004.

[5] A. Shlivinski and K. J. Langenberg. Defect imaging with elastic waves in inhomogeneous-anisotropic materials with composite geometries. Ultrasonics, 46(1): 89-104, 2007.

[6] Q. M. Tai and M. H. Sadd. A discrete element study of the relationship of fabric to wave propagational behaviours in granular materials. Int. J. for Num. and Analytic meth. in Geomechanics, 21(5): 295-311, 1997.

[7] M. H. Sadd, G. Adhikari, F. Cardoso. DEM simulation of wave propagation in granular materials. Powder Technology, 109(1-3): 222-233, 2000.

[8] S. Luding. Granular media: Information propagation. Nature, 435:159-160, 2005 .

[9] V. Tournat, B. Castagnede, V. Gusev and P. Bequin. Self-demodulation acoustic signatures for nonlinear propagation in glass beads. Comptes rendus Mecaniques, 331(2):119-125, 2003.

[10] V. F. Nesterenko. Dynamics of Heterogeneous Materials. Springer-verlag New York, 2001.

[11] N. Dejong, L. Hoff, T. Skotland, et al. Absorption and scatter of encapsulated gas filled microspheres - Theoretical considerations and some measurements. Ultrasonics, 30(2): 95-103, 1992.

[12] X. Jia, C. Caroli, and B. Velicky. Ultrasound propagation in externally stressed granular media. Phys. Rev. Lett., 82(9):1863, 1999.

[13] S. Luding. Micro-macro transition for anisotropic, frictional granular packings. Int. J. Sol. Struct., 41:5821-5836, 2004.

[14] I. Agnolin, J. T. Jenkins and L. La Ragione A continuum theory for a random array of identical, elastic, frictional disks. Mechanics of Materials, 38(8-10):687701, 2006.

[15] J. Jenkins, D. Johnson, L. La Ragione, et al. Fluctuations and the effective moduli of an isotropic, random aggregate of identical, frictionless spheres. Journal of the Mechanics and Physics of Solids, 53(1):197-225, 2005. 
[16] V. Tournat, private communication, unpublished, 2007.

[17] S. Luding. Cohesive frictional powders: Contact models for tension. Granular Matter, in press, 2007.

[18] P. A. Cundall and O. D. L. Strack. A discrete numerical model for granular assemblies. Géotechnique, 29(1):47-65, 1979.

[19] O. Mouraille and S. Luding. Sound propagation in dense, frictional granular materials. In R. Garcia-Rojo, H. J. Herrmann, and S. McNamara, editors, Powders and Grains 2005, page 319, Leiden, 2005. A. A. Balkema Publishers.

[20] O. Mouraille, W. A. Mulder and S. Luding. Sound wave acceleration in granular materials J. Stat. Mech., P07023, 2006.

[21] O. Mouraille and S. Luding. Mechanic waves in sand: Effect of polydispersity In W. Peukert, editor, Partec 2007, CD proceeding, 2007.

[22] S. Luding. Stress distribution in static two-dimensional granular model media in the absence of friction. Phys. Rev. E, 55:4720-4729, 1997.

[23] E. Somfai, J-N. Roux, J. H. Snoeijer, M. van Hecke, and W. van Saarloos. Elastic wave propagation in confined granular systems. Phys. Rev. E, 72(2):021301, 2005 .

[24] Q. M. Tai and M. H. Sadd Contact law effects on wave-propagation in particulate material using distinct element modeling. Int. J. Non-Linear Mech. 28(2): 251-265, 1993.

[25] V. Tournat, et al. Probing weak forces in granular media through nonlinear dynamic dilatancy: clapping contacts and polarization anisotropy. Phys. Rev. Lett., 92(8):085502, 2004. 